\title{
Experiments and Evaluation Based Pixellated CZT Semiconductor Detector
}

\author{
Shen Min ${ }^{1,2, a}$, Tang Zhi-ling ${ }^{1, b}$ \\ ${ }^{1}$ Chongqing Technology and business institute, Chongqing hechuan,401520, China \\ ${ }^{2}$ The Key Laboratory of Optoelectronic Technology and system, Ministry of Eduacation, \\ Chongqing University, Chongqing 400030, China \\ aEmail:Simi_xuan@126.com,simi_xuan@yahoo.com.cn \\ b Email: 937122274@qq.com
}

Keyword: Cadmium zinc telluride, semiconductor detector, Indium contacts, dark current

\begin{abstract}
Cadmium zinc telluride (CZT) material is one of the preferred materials for the fabrication of X-ray and gamma-ray detector. In this paper, it is presented an experimental detector system based on pixellated CZT semiconductor detector. At the same time, some research and design on the surface signal-readout method and the preamplifier circuitry is made. The signal coming from the CdZnTe material exposed to the radiation through the experiment is successfully required. The collection-efficiency between the electron and the hole in the anode is test when the different bias is applied in the pixellated CZT semiconductor detector. The parameter of the CZT detector and validate the responsive effect for the radiation is evaluated.The experiments and evaluation basis for the development of subsequent gamma spectrometer.
\end{abstract}

\section{Introduction}

In recent years, the world wide effort to fight against nuclear and radiation terrorism has caused the urgent demand for $\gamma$-spectrometers that can work under the room temperature .CdZnTe (CZT) is very attractive material for room-temperature semiconductor detectors due to its wide bandgap and high atomic number ${ }^{[1]}$. The CZT detector has a great advantage to have the high density $\left(\left(\rho=5: 86 \mathrm{~g} / \mathrm{cm}^{-3}\right)\right.$ and the high atomic number $(Z=48$ for $\mathrm{Cd}, 30$ for $\mathrm{Zn}$ and 52 for $\mathrm{Te})$ compared with Si $\left(\rho=2.4 \mathrm{~g} / \mathrm{cm}^{-3}, Z=14\right)$ and $\mathrm{Ge}\left(\rho=5.36 \mathrm{~g} / \mathrm{cm}^{-3}, Z=32\right)$ semiconductor detectors. Nowadays a larger crystal size of CZT becomes available and the CZT detector can be used for higher energy gamma ray detection ${ }^{[2]}$. However, holes of CZT detectors have poor mobility. Thus, to achieve high-energy resolution and satisfy imaging demand, the pixellated CZT detector is applied in our experiment.

IMARAD Imaging Systems mostly manufacture CZT detector that apply to the medicine diagnose. In this situation, the CZT detector mostly response low-energy radiation. In this paper, we apply the CZT detector system that respond the energy under $200 \mathrm{keV}$ to the high-energy detection. The results from testing different condition are reported, which help us to characterize the performance and determine the way of our subsequent independent research on the CZT detector .Finally, we will achieve the CZT imaging system through making use of the existing CZT detector system.

\section{Experiment Setup}

\section{Detector Module}

We used CZT detector manufactured by IMARAD Imaging Systems, Israel for our study. IMARAD CZT crystals are grown by the modified Horizontal Bridgman method. It is a known fact that IMARAD CZT material is slightly N-type material. The crystal properties such as resistivity depend on the metallic contact. Typical IMARAD CZT crystal comes in $38.92 \times 38.92 \times 5 \mathrm{~mm}$ size and with Indium contacts on both anode and cathode surface. The anode surface is pixelated in $16 \mathrm{x}$ 16 array of pixels with pitch of $2.46 \mathrm{~mm}$. Typical gap between pixel pads is $0.6 \mathrm{~mm}^{[3]}$. The floating 
guard band surround the edge pixels. Pixel dark current of the crystal is $20 \mathrm{nA}$. The detector system usually adapt to detecting several $\mathrm{keV} \sim 200 \mathrm{keV}$ radiation. Through adjusting some parameter of the system, the module can be attempted to detect high-energy.

\section{Evaluation System}

The system mostly include three parts: readout system, signal processing and control, analysis and display platform. The CZT detector array have several CZT crystals and two crystals have $8 \times 8$ pixels and another one have $16 \times 8$ pixels .The readout circuit under every pixel get the pixel signal ,amplify and shaper the signal, finally send to multi-channel analyzer based on FPGA board .PC display the spectrum of every pixel signal via USB port ${ }^{[4]}$. We analyze the spectrum and can determine characteristic spectral line of the radiation .Furtherly, the radiant substance can be evaluated through the characteristic spectral line. The block diagram of the whole evaluation system is show in fig. 1.

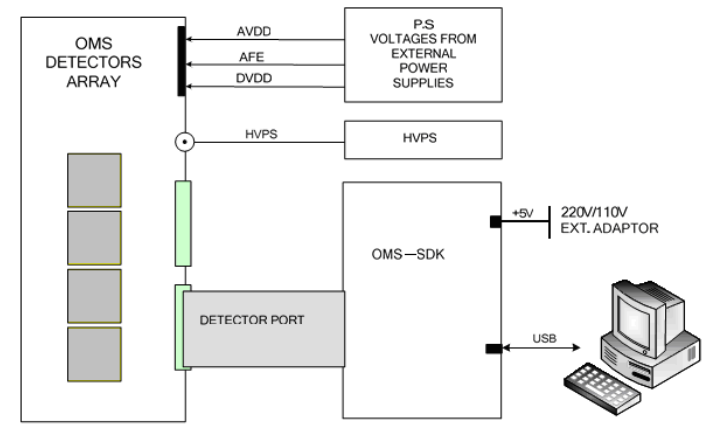

Fig.1 The block diagram of the evaluation system

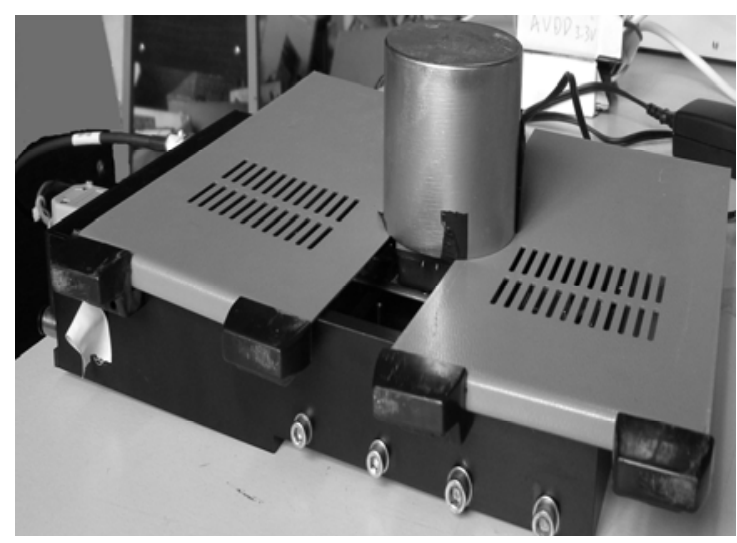

Fig2 The laboratorial system that is used to radiation properties measurement

\section{Radiation properties measurements}

Our radiation properties measurement setup is based on the CZT detector manufactured by IMARAD Imaging Systems. The radiation source is placed at different region. During the research , ${ }^{60} \mathrm{Co}$ and ${ }^{137} \mathrm{Cs}$ are the $\gamma$-ray sources that we use. The respective characteristic spectral line is 122 $\mathrm{keV}$ and $662 \mathrm{keV}$. The low energy threshold of our system is approximately $20 \mathrm{keV}$. Of course, the threshold can be changed by the software according to the specific situation .The laboratorial system is show in the fig. 2 .

\section{Results}

We carry out a series of experiments at different conditions and get many results. We will detailed describe the results in the following text.Fig. 3 show the result that the $\gamma$-ray source is not applied. From the figure, almost all pixels are not response in this condition except the several left-edge pixels. This phenomenon may be caused by the leakage current. When the experiment is being, the outside circumstance certainly will work the CZT crystal especial for the edge pixels. 
In the next experiment, the $\gamma$-ray source is used to the system . When the system is applied $400 \mathrm{~V}$ bias and acquisition time is 100 second. The distance is $15 \mathrm{~cm}$ between the source and the CZT crystal. Fig. 4 show the result under this term. If the bias is positive, the anode electrode

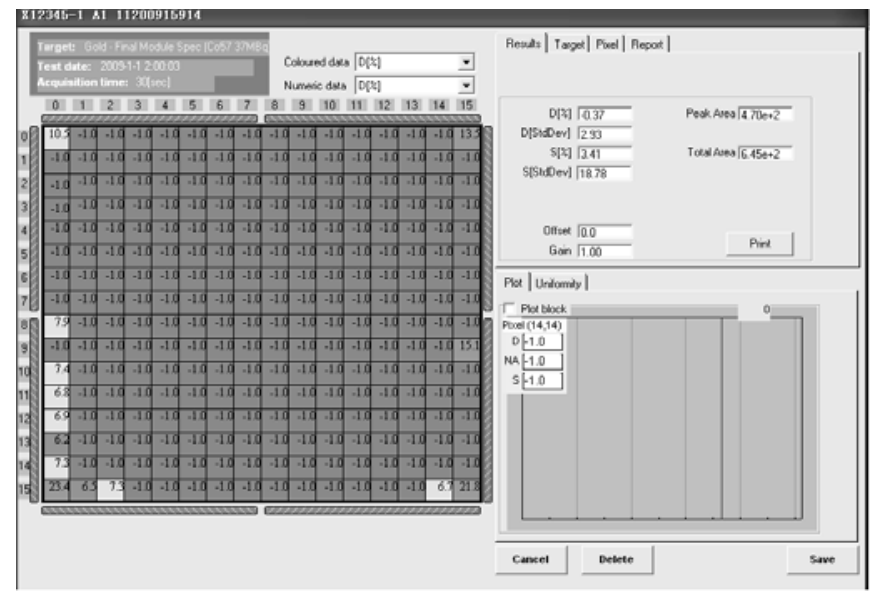

Fig.3 The pixel detection-result of the CZT array are not exposed to $\gamma$-ray source.

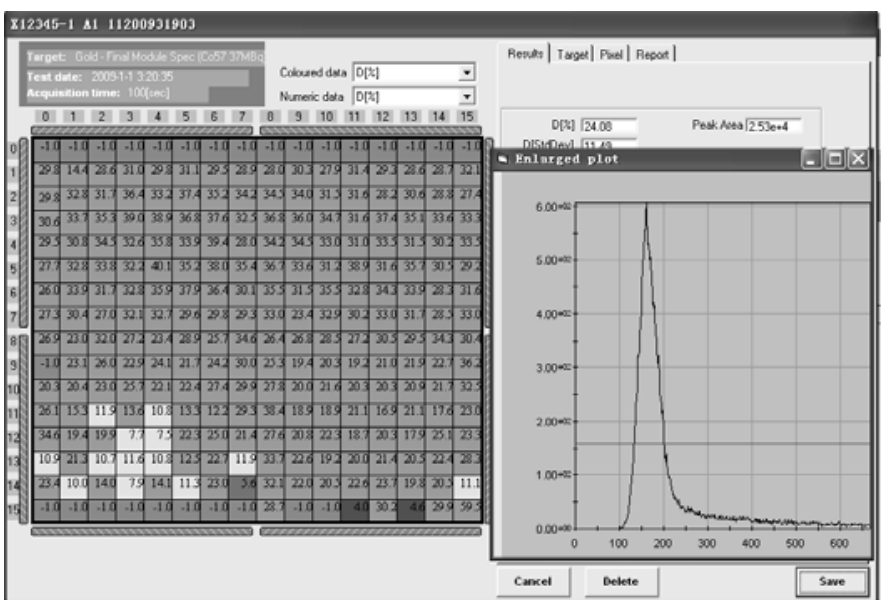

Fig.4 The pixel detection-result of the CZT array are exposed to $\gamma$-ray source while the bias is $400 \mathrm{~V}$ and the acquisition time is 100 second .

mostly collect holes. However, holes of CZT detectors have poor mobility. So in the spectrum ,the low-energy tail aroused by the hole-trapping is obviously ${ }^{[5]}$. Every pixel has different energy resolution. But most pixels are not ideal. The best result of the energy resolution is $7.5 \%$.

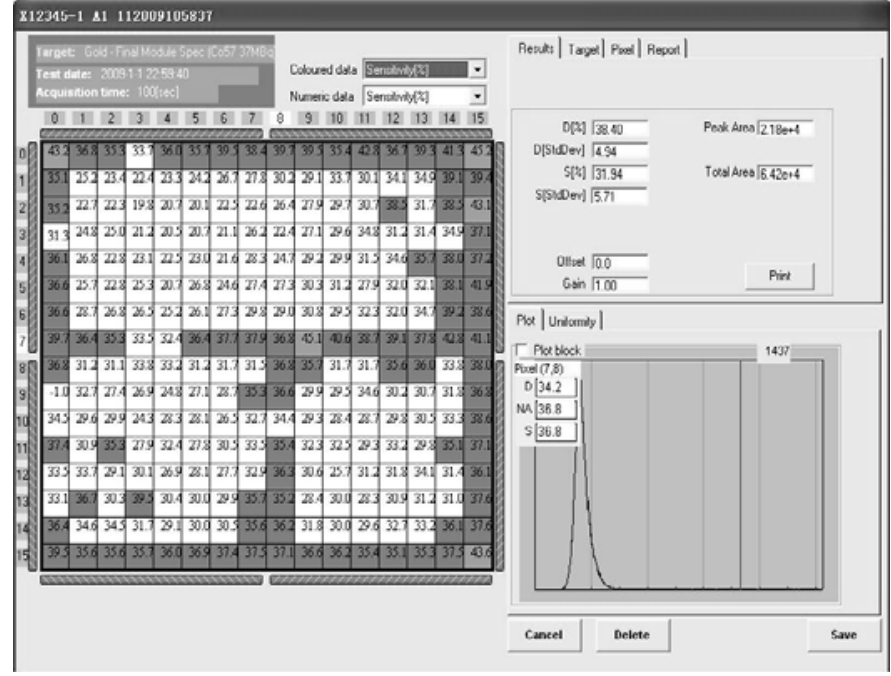

Fig.5 The result when the bias is $-900 \mathrm{~V}$ and the acquisition time is 100 second

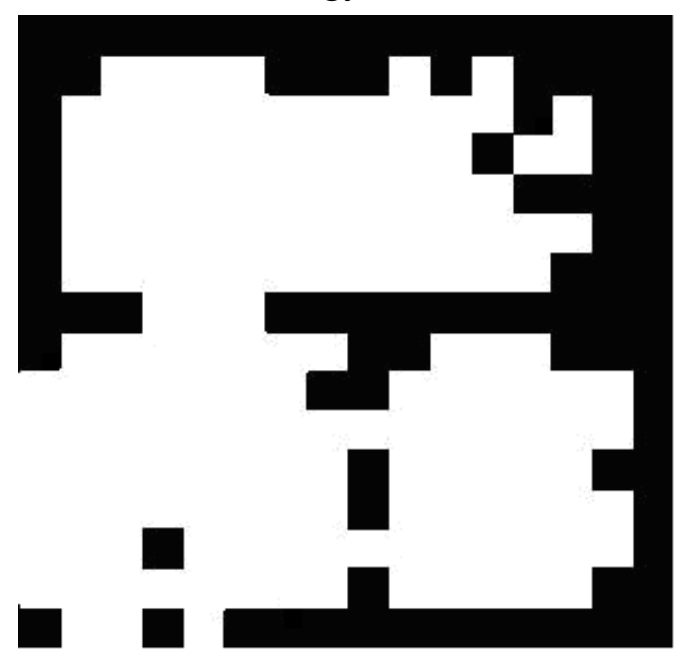

Fig.6 The binary image of the sensitivity

When the system is applied $-900 \mathrm{~V}$ bias and acquisition time is 100 second. The source is placed on the interface that cover the lower right region of the crystal. The Fig. 5 show the result under this term. If the bias is negative, the anode electrode mostly collect electrons. Because the electron of CZT detectors have good mobility ${ }^{[6]}$. So in the spectrum ,the low-energy tail is not obviously.

We transform the result into the binary image that can preferably display the pixel sensitivity . Fig.6 show the processed result .From the binary image, we can see that pixels of the lower right have better sensitivity. This correspond with the theoretical result.

\section{Discussions}

We carried out a comparative study of the system . From the study, we can make a conclusion 
that the whole system has not a good response for the $\gamma$-ray source. The energy resolution is not so good. There are below several reasons that induce our experiment is not ideal.

First: the radiant intensity of the source is not enough. The radiant intensity is millicurie when the test is being. The little radiant intensity is often submerged in outside interference such as light, noise coming from other instrument and so on.

Second: the bias that applied is not so stabile. That will bring the collect-field fluctuant not in favor of the collection of the carriers.

We will sum up the experience during the research .In the future, some measurement will be used to improve the whole system. We hope we will make progress in the energy resolution and the performance of the system.

\section{Acknowledgement}

It is a project supported by the National Natural Science Foundation（10876044/A06）.

\section{Reference:}

[1] T. E. Schlesinger and R. B. James, Semiconductors and Semimetals, in Semiconductors for Room Temperature Nuclear Detector Applications, Vol. 43, San Diego, CA Academic, 1995.

[2]A.H.D. Rasolonjatovo et al, Development of Gamma Ray Monitor Using CdZnTe Semiconductor Detector, Proceedings of the Second International Workshop on EGS, 8.-12. August 2000, Tsukuba, Japan KEK Proceedings 200-20, pp.144-151

[3] S.V. Vadawale et al, Multipixel characterization of imaging CZT detectors for hard X-ray imaging and spectroscopy, Send correspondence to S. V. Vadawale

[4]Y. Nemirovsky et al., Recent progress in n-type CdZnTe arrays for gamma-ray spectroscopy, NIM A, V. 458 1-2, p.325, (2001)

[5] L.Verger et al ,characterization of CdTe and CdZnTe detectors for gamma-ray imaging application,Nuclear Instrument and Methods in Physics Research A 458(2001)297-309

[6]H.Hermon et al, CZT detector fabricated from horizontal and vertical Bridgman-grown crystals, Nuclear Instrument and Methods in Physics Research A 458(2001)503-510 